\title{
$\gamma$ detectors for Deep Inelastic Neutron Scattering in the 1-100 eV energy region
}

\author{
A. Pietropaolo ${ }^{1, *}$, C. Andreani ${ }^{1}$, A. D’Angelo ${ }^{2}$, R. Senesi ${ }^{3}$, G. Gorini ${ }^{4}$, S. Imberti ${ }^{4}$, M. Tardocchi ${ }^{4}$, N. Rhodes ${ }^{5}$, \\ E.S. Schooneveld ${ }^{5}$ \\ ${ }^{1}$ Dip. di Fisica, Universita' degli Studi di Roma Tor Vergata e INFM, via della Ricerca Scientifica 1, 00133 Roma, Italy \\ ${ }^{2}$ Dip. di Fisica, Universita' degli Studi di Roma Tor Vergata e INFN, via della Ricerca Scientifica 1, 00133 Roma, Italy \\ ${ }^{3}$ INFM e Universita' degli Studi di Roma Tor Vergata, via della Ricerca Scientifica 1, 00133 Roma, Italy \\ ${ }^{4}$ Dipartimento di Fisica “G. Occhialini”, Universita' degli Studi di Milano-Bicocca e INFM, Edificio U2, Piazza delle Scienze 3, 20126 Milano, Italy \\ ${ }^{5}$ Rutherford Appleton Laboratory, Chilton, Didcot, Oxfordshire, OX11 0QX, UK
}

Received: 23 July 2001/Accepted: 24 October 2001 - @ Springer-Verlag 2002

\begin{abstract}
The Resonant Detector Technique (RDT) is a very promising technique for neutron spectroscopy in the epithermal energy region above $1 \mathrm{eV}$ at spallation neutron sources. In this technique, using the $(n, \gamma)$ reactions, the energy of the scattered neutrons is assigned by revealing the prompt $\gamma$ ray cascade from the absorbing foil through a $\gamma$ detector. The advantages of this technique, as compared to the Single Difference Technique (SDT) currently used on eVS spectrometer at ISIS, are presented. Different choices of converter foils and $\gamma$ detectors will be compared and discussed, especially in terms of their efficiency and background insensitivity.
\end{abstract}

PACS: 61.12.-q; 61.12.Ex

In the last few years the high flux of neutrons above $1 \mathrm{eV}$, available at the ISIS spallation neutron source, has extended the kinematic region at high momentum ( $q$ up to $200 \AA^{-1}$ ) and energy ( $\hbar \omega$ up to $10 \mathrm{eV}$ ) transfers for spectroscopy studies in condensed matter [1]. Deep Inelastic Neutron Scattering (DINS) measurements with epithermal neutrons are routinely performed with the eVS spectrometer at the ISIS facility, using the Single Difference Technique (SDT) [2,3]. In the latter the final energy of the scattered neutrons is fixed by an absorbing foil (typically ${ }^{197} \mathrm{Au}$ or ${ }^{238} \mathrm{U}$ ), the initial energy being determined by the time-of-flight technique. During measurements, the foil is cycled in and out of the scattered neutron beam. The intensity of neutrons scattered with final energy $E_{1}$ is calculated by performing the difference between the spectra acquired with and without the absorber. This technique, by employing ${ }^{6} \mathrm{Li}$-glass scintillators as neutron detectors, allows access to energy transfers, $\hbar \omega$, in the $1-30 \mathrm{eV}$ range and wavevector transfers, $q$, in the $30-200 \AA^{-1}$ range. An upgrade of the eVS spectrometer is the recently installed VESUVIO instrument [4], which by employing the Double Difference Technique (DDT) [5] will improve considerably the instrumental resolution. In this case the scattered signal is obtained by a weighted difference between the signals

*Corresponding author.

(Fax: +39-06/2023507, E-mail: antonino.pietropaolo@ roma2.infn.it) coming from two resonant foils of the same material but of different thickness [6]. In order to perform epithermal neutron scattering in a wider kinematic space (i.e. $q \leq 10 \AA^{-1}$ coupled to $\hbar \omega \geq 1 \mathrm{eV}$ and $q \geq 200 \AA^{-1}$ coupled to $\left.\hbar \omega \geq 1 \mathrm{eV}\right)$, it is necessary to explore a different approach. In this context a promising technique is the Resonant Detector Technique (RDT) [7]. This involves the detection of the prompt $\gamma$ radiation cascade emitted by the absorber foil after resonant neutron capture. As it has recently been shown [8], the advantages are twofold. Firstly, the experimental signal can be obtained as a direct measurement, rather than by a difference between foil-in and foil-out spectra. Secondly, $\gamma$ detectors for high-energy neutron scattering do not suffer from the poor efficiency and excessive background rates of the ${ }^{6} \mathrm{Li}$-glass scintillation detectors. Furthermore, using the $\gamma$ detectors coupled with the DDT, there is no need of foil-out subtraction, and instrumental resolution can be improved with good statistical data [8].

\section{Resonant absorbers}

In the RDT approach the choice of resonant foils to be used for scattered neutron absorption in DINS measurements is a crucial step. The cross-section of the resonance foil has to have well-isolated and intense peaks in the $1-100 \mathrm{eV}$ region with narrow widths (FWHM) compared to the resonance energy. The material itself requires a high yield of low-energy $\gamma$ radiation. The first requirement is very important in order to identify and analyze the recoil peaks reliably and to lower the $\Delta E_{\mathrm{r}} / E_{\mathrm{r}}\left(E_{\mathrm{r}}\right.$ being the energy resonance). This ensures a low contribution to the energetic component of the spectrometer resolution function. The second requirement is related to the possibility of using $\gamma$ detectors with a small effective volume and good efficiency for low-energy $\gamma$ radiation. In this context an investigation of possible absorbers, meeting the characteristics mentioned above, is reported in Table 1. All these elements emit $\gamma$ radiation with appreciable relative intensities, $I_{\mathrm{r}}$, in the $10-300 \mathrm{keV}$ region [9]. This is in the energy range of interest to test different detectors referred to in the next section. 
Table 1. List of absorbers to be tested in DINS experiments using the Resonant Detector Technique. $E_{\mathrm{r}}$ is expressed in $\mathrm{eV}, \sigma$ in barns and $\Gamma$ in $\mathrm{meV}$

\begin{tabular}{lrrr}
\hline Isotope & $E_{\mathrm{r}}$ & $\sigma$ & $\Gamma$ \\
& & & \\
\hline${ }^{113} \mathrm{In}_{49}$ & 14.6 & 9965 & 67 \\
${ }^{139} \mathrm{La}_{57}$ & 72.2 & 5969 & 96 \\
${ }^{150} \mathrm{Sm}_{62}$ & 20.7 & 56207 & 109 \\
${ }^{160} \mathrm{Dy}_{66}$ & 20.5 & 16165 & 124 \\
${ }^{168} \mathrm{Er}_{68}$ & 79.7 & 11203 & 121 \\
${ }^{178} \mathrm{Hf}_{172}$ & 72.6 & 16838 & 112 \\
${ }^{182} \mathrm{~W}_{74}$ & 21.1 & 46800 & 104 \\
${ }^{190} \mathrm{Oss}_{76}$ & 91.0 & 6777 & 105 \\
${ }^{238} \mathrm{U}_{92}$ & 6.67 & 23564 & 25 \\
${ }^{238} \mathrm{U}_{92}$ & 20.9 & 37966 & 57 \\
${ }^{238} \mathrm{U}_{92}$ & 36.7 & 42228 & 48 \\
${ }^{238} \mathrm{U}_{92}$ & 66.0 & 20134 & 139 \\
${ }^{197} \mathrm{Au}_{79}$ & 4.9 & 36592 & \\
\hline
\end{tabular}

\section{Detectors}

The RDT coupled to the DDT has been successfully applied in DINS measurements [8] using a ${ }^{238} \mathrm{U}$ absorber and a $3^{\prime \prime} \times 3^{\prime \prime} \mathrm{NaI}(\mathrm{Tl})$ scintillator. In spite of the encouraging and important results, the signal-to-noise ratio is still not satisfactory, since the large volume of the device give rise to a high background sensitivity. Previous results have to be considered as a good guide to the final choice of suitable detectors. Valid ones are the HpGe, the silicon detectors and the new solid-state detectors, the so-called CZT $\left(\mathrm{Cd}_{1-x} \mathrm{Zn}_{x} \mathrm{Te}\right)$. All these devices show good efficiency and resolution in the $\gamma$ energy range of interest; but different tests have to be performed in order to collect precise indications of their possible use in DINS measurements. The HpGe has excellent resolution (0.003 FWHM at $122 \mathrm{keV})$ allowing $\gamma$ discrimination, but it suffers from radiation damage by fast neutrons. Furthermore, it needs to be cooled to a low temperature to minimize leakage current effects. Silicon detectors have a worse energy resolution $(\leq 0.04 \mathrm{FWHM}$ at $59.5 \mathrm{keV})$. With respect to $\mathrm{HpGe}$, they have a high efficiency for $\gamma$ radiation up to $30 \mathrm{keV}$ energy, they can work at room temperature and they are more resistant to fast neutron damage. The CZT detectors are new devices: they are compact (commercially available devices have sizes ranging from $5 \mathrm{~mm} \times 5 \mathrm{~mm} \times 1 \mathrm{~mm}$ to $10 \mathrm{~mm} \times 10 \mathrm{~mm} \times 2 \mathrm{~mm})$ and they have good energy resolution $(\simeq 0.06 \mathrm{FWHM}$ at $59.5 \mathrm{keV})$ [10]. No information is available regarding the degree of damage due to neutrons. The presence of cadmium in the device could contribute significantly to the background due to the high thermal neutron absorption cross-section of cadmium. The routine use of $\gamma$ detectors and the development of a very-low-angle array of detectors will allow new experimental studies in condensed matter experiments with low- $q$ and high-energy transfers to be performed. To this aim, tests on the whole set of detectors discussed above are planned shortly at the ISIS pulsed neutron source on the VESUVIO spectrometer.

\section{References}

1. J. Mayers: Phys. Rev. B 41, 41 (1990)

2. C. Andreani, A. Filabozzi, M. Nardone, F.P. Ricci, J. Mayers: Phys. Rev. B 50, 12744 (1994); J. Mayers, A.C. Evans: RAL Report RAL91-048 (Rutherford Appleton Laboratory, Chilton, Didcot, Oxfordshire, UK 1991)

3. D. Colognesi, C. Andreani, R. Senesi: Europhys. Lett. 50, 202 (2000)

4. R. Senesi, C. Andreani, Z. Bowden, D. Colognesi, E. Degiorgi, A.L. Fielding, J. Mayers, M. Nardone, J. Norris, M. Praitano, N.J. Rhodes, W.G. Stirling, J. Tomkinson, C. Uden: Physica B 276-278, 200 (2000)

5. C. Andreani, E. Degiorgi, A. Filabozzi, D. Colognesi, M. Nardone: Internal Report Rom2F/98/04 (1998)

6. P.A. Seeger, A.D. Taylor, R.M. Brugger: Nucl. Instrum. Methods A 240, 98 (1985)

7. R.G. Johnson: Nucl. Instrum. Methods Phys. Res., Sect. A 263, 427 (1988); N. Watanabe: Report IAEA-CN-46/26 (1985) p. 279

8. C. Andreani, A. Pietropaolo, R. Senesi, G. Gorini, M. Tardocchi, A. Bracco, N. Rhodes. E.M. Schoonefeld: Nucl. Instrum. Methods Phys. Res., Sect. A, in press (2002)

9. J.K. Tuli: Thermal Neutron Capture Gamma-rays (National Nuclear Data Center, Brookhaven National Laboratory, Upton, N.Y. 1999); available at http://www.nndc.bnl.gov/wallet/tnc/capgam.shtml

10. D.S. McGregor, H. Hermon: Nucl. Instrum. Methods A 395, 101 (1997) 